\title{
New theories in endometriosis: new knowlage may be producing novel therapies
}

Dr. Laila Ezzat, MD

Obstetrics and gynecology department, Aswan University, Aswan, Egypt
Corresponding Author:

Dr. Laila Ezzat

Obstetrics and gynecology

department, Aswan University,

Aswan, Egyp

mobile: +201117234776

E-mail address: lailaezzat972000@

gmail.com

\section{$\underline{\text { Abstract }}$}

Endometriosis is a common, chronic inflammatory disease defined by the presence of extrauterine endometrial tissue. The aetiology of endometriosis is complex and multifactorial, where several not fully confirmed theories describe its pathogenesis.

The current literature suggests that stem cells, dysfunctional immune response, genetic predisposition, and aberrant peritoneal environment may all be involved in the establishment and propagation of endometriotic lesions. An orchestrated scientific and clinical effort is needed to consider all factors involved in the pathogenesis of this multifaceted disease and to propose novel therapeutic targets to reach effective treatments for this distressing condition.

\section{Introduction}

Endometriosis is a chronic, benign, oestrogen-dependent inflammatory disease affecting approximately $10 \%$ of reproductive age women and $35-$ $50 \%$ of women with pelvic pain and infertility [ 1]

It can be a debilitating disease with symptoms of dysmenorrhoea, dyspareunia, and chronic pelvic pain [2].

The definition of endometriosis is histological and it requires the identification of the presence of endometrial gland and stroma-like tissue outside (ectopic) the uterus. These ectopic lesions are commonly located on the pelvic organs and peritoneum [3]

Occasionally, ectopic endometriotic lesions can be found in other parts of the body such as kidney, bladder, lungs, and even in the brain [4].

The clinical presentation of endometriosis is varied and conclusive diagnosis requires laparoscopy [5].

There has been efforts to standardize the surgical staging of endometriosis and histopathological changes with updated modified American Fertility Society scoring [6].

However this objective surgical staging does not necessarily correlate with the clinical symptoms[7].

Furthermore, there is a severe lack of knowledge on the natural progression of the disease in women since the severity measurement will require repeated invasive surgery. There are reports of endometriosis associated with spontaneous regression, no progression [8].

and progressing to ovarian carcinomas [9].

The oldest theory, that of metaplasia, suggests that under diverse influences coelomic tissue could be transformed into endometrium. The most often cited theory, that of implantation, proposes that the physiological phenomenon of endometrial reflux in the fallopian tubes during 
menstruation may, in certain conditions, overcome local defense mechanisms, implant, and proliferate[10]

Endometriosis is estimated to occur in roughly $6-10 \%$ of women[11].

It is most common in those in their thirties and forties; however, can begin in girls as early as 8 years old[12].

It results in few deaths with this being estimated at 200 globally in 2013.[13].

Endometriosis was first determined to be a separate condition in the 1920s. Before that time endometriosis and adenomyosis were considered together. It is unclear who first described the disease.[14].

Endometriosis was first discovered microscopically by Karl von Rokitansky in 1860 [15].

although it was documented in medical texts more than 4,000 years ago.[16].

The Hippocratic Corpus outlines symptoms similar to endometriosis, including uterine ulcers, adhesions, and infertility.[16].

\section{Methods:}

\section{Search Strategy and Selection Criteria}

Initially searched "Pubmed" for relevant literature using the terms "endometriosis" and "pathogenesis" or "classification" for studies published up to 2016 . Although those papers provided the basis for this review, for detailed understanding of the topic we extended the search to much older yet frequently referred articles. Studies that were deemed suitable by the authors included those that examined the pathophysiology of human endometriosis: from in vitro basic science (molecular, genetical and functional) studies, studies employing animal (rodent/primate) models, gene expression, and epidemiological studies.

\section{$\underline{\text { Results }}$}

\section{Classification of Endometriosis}

Interrogation of pathogenesis of endometriosis highlights the current drawbacks associated with the classification of this disease. The revised American fertility society classifies endometriosis according to multiple criteria including histopathological as well as anatomical features, distinguishing superficial endometriosis from deep lesions of the peritoneum and ovaries[17].

Deep endometriosis is defined arbitrarily as adenomyosis externa, infiltrating the peritoneum by $>5 \mathrm{~mm}[18]$.
It is noteworthy that the current classification system is limited by observer error as well as reproducibility and this may explain the poor correlation between extent of the disease and its clinical presentation [7].

Furthermore, histological information of endometriosis is limited by the technical efficiency in endometriotic biopsy sampling and processing, particularly when the lesions are located close to organs such as ureters, bowel and bladder [5]. A separate classification system (ENZIAN score) has been recently introduced for deep infiltrating endometriosis. It is a helpful aid in describing this type of endometriosis but it needs further refinement [19].

Superficial and deep endometriosis has been categorised by some authors as two different diseases with different pathogeneses whereas others regard them as different manifestations of the same disease. Naturally this lack of consensus with disease classification creates another ambiguity around much of the available literature on pathogenesis.[19].

Theories on the Pathogenesis of Endometriosis

\section{Retrograde Menstruation}

It suggests that during a woman's menstrual flow, some of the endometrial debris exits the uterus through the fallopian tubes and attaches itself to the peritoneal surface (the lining of the abdominal cavity) where it can proceed to invade the tissue as endometriosis.[20].

In non-human primate models, it is possible to induce endometriosis by inoculating autologous menstrual products simulating retrograde menstruation in the peritoneal cavity of baboons and macaques[22].

With a single inoculation of menstrual endometrial tissue directly in to the pelvic cavity, up to $46 \%$ of the animals have shown development of endometriotic lesions in the pelvic cavity [23].

whereas $100 \%$ of animals developed peritoneal endometriotic lesions after two consecutive cycles of inoculations of curetted menstrual endometrium. These lesions were histologically and clinically similar to human ectopic endometriotic lesions[24].

However, retrograde menstruation occurs in 76\%-90\% of women with patent fallopian tubes and not all of these women have endometriosis[21].

Retrograde menstruation alone is not able to explain all instances of endometriosis, and additional factors such as genetic or immune differences need to be invoked to account for the fact that many women with retrograde menstruation do not have endometriosis. Researchers 
are investigating the possibility that the immune system may not be able to cope with the cyclic onslaught of retrograde menstrual fluid. In this context there is interest in studying the relationship of endometriosis to autoimmune disease, allergic reactions, and the impact of toxic materials[25][26].

This theory has been disputed in the past since it cannot explain the occurrence of endometriosis in prepubertal girls, newborns, or males [27].

\section{Metaplasia}

The Coelomic metaplasia theory postulates that endometriosis originates from the metaplasia of specialised cells that are present in the mesothelial lining of the visceral and abdominal peritoneum [28].

Coelomic cells which are the common ancestor of endometrial and peritoneal cells may undergo metaplasia from one type of cell to the other, perhaps triggered by inflammation.[29].

The coelomic metaplasia theory may explain the occurrence of endometriosis in prepubertal girls [30].

However, the usual driving force for endometrial growth, oestrogen, is not present in the pre-pubertal girls and therefore this condition may be different from endometriosis that is found in women of reproductive age [3].

\section{Müllerianosis}

A theory supported by foetal autopsy is that cells with the potential to become endometrial, which are laid down in tracts during embryonic development called the female reproductive (Mullerian) tract as it migrates downward at 8-10 weeks of embryonic life, could become dislocated from the migrating uterus and act like seeds or stem cells.[31].

\section{Environment; Environmental toxins}

(e.g.; dioxin, nickel) may cause endometriosis.[32].

\section{Vasculogenesis:}

Up to $37 \%$ of the microvascular endothelium of ectopic endometrial tissue originates from endothelial progenitor cells(EPCs), which result in de novo formation of microvessels by the process of vasculogenesis rather than the conventional process of angiogenesis[33].

Angiogenesis is defined as the formation of new microvessels from pre-existing ones and occurs via sprouting angiogenesis and intussusception.[34].
Vasculogenesis was originally defined as de novo blood vessel formation by migration and differentiation of angioblastic progenitor cells during embryonic and fetal development[35].

it is now well accepted that vasculogenesis also contributes to the formation of new blood vessels in the adult. This so-called post-natal vasculogenesis is characterized by the mobilization of bone marrowderived or tissue-resident EPCs into the bloodstream in response to certain cytokines or tissue ischaemia. These circulating EPCs are then recruited into sites of neovascularization, where they are incorporated into the vascular endothelial lining and differentiate in situ into endothelial cells[36].

Process, termed post-natal vasculogenesis, is an integral part of the pathogenesis of endometriosis, which may have important implications for the establishment of novel diagnostic and therapeutic strategies for this frequent gynaecological disease.[33].

\section{Neural growth}

An increased expression of new nerve fibres is found in endometriosis, but does not fully explain the formation of ectopic endometrial tissue, and is not definitely correlated with the amount of perceived pain [37].

\section{Hormones}

Steroid hormones should play a central role in the aetiology of endometriosis since it is a disease of women in reproductive age and not usually seen in postmenopausal women who are not on hormonal treatment [38].

Environmental toxins, such as dioxin, are implicated in the aetiology of endometriosis, which may mimic oestrogen via interacting with oestrogen receptors[38].

Furthermore, there may be a higher bioavailability of oestradiol in endometriotic tissue due to the local aromatisation of circulating androgens to oestradiol by endometriotic stromal cells and also there may be reduced conversion of oestradiol to the less potent oestrone due to the ectopic endometriotic tissue expressing decreased 17ß-hydroxysteroid enzymes [3].

\section{Oxidative Stress and Inflammation}

Increased oxidation of lipoproteins has been associated with the pathogenesis of endometriosis, where reactive oxygen species (ROS) cause lipid peroxidation that leads to DNA damage in endometrial cells [39].

The presence of water and electrolytes in the increased peritoneal fluid volume in patients with endometriosis 
harbours the source of ROS [40].

These patients also have iron overload in their peritoneal cavities from the breakdown of haemoglobin, which in turn causes redox reactions [41].

The release of the proinflammatory heam products and the oxidative stress signals generated from the ROS cause inflammation which leads to the recruitment of lymphocytes and activated macrophages producing cytokines that induce oxidizing of enzymes and promotes endothelial growth [42].

\section{Immune Dysfunction}

Graves disease is an autoimmune disease characterized by hyperthyroidism, goiter, ophthalmopathy, and dermopathy. Women with endometriosis had higher rates of Graves disease. One of these potential links between Graves disease and endometriosis is autoimmunity[43].

Women with endometriosis have a higher concentration of activated macrophages, decreased cellular immunity, and a repressed natural killer(NK cell) function [44] [45].

Possibly as a consequence, women with endometriosis have higher expression of cytokines and vascular endothelial growth factors in their peritoneal fluid, which promote proliferation of endometrial cells and angiogenesis[46].

\section{Apoptosis Suppression and Alteration of Endometrial Cell Fate}

Alteration of the endometrial cell fate to favour antiapoptotic and proproliferative phenotype is paramount for the survival of the endometrial cells in the peritoneal cavity to initiate ectopic deposits and for the maintenance of the established lesions [47].

By examining matched eutopic endometrium and ectopic lesions from women with endometriosis and in baboon with induced disease, we have recently shown that telomerase enzyme may play a central role in this altered endometrial cell phenotype [47].

By examining matched eutopic endometrium and ectopic lesions from women with endometriosis and in baboon with induced disease, we have recently shown that telomerase enzyme may play a central role in this altered endometrial cell phenotype [47].

There is plethora of evidence suggesting an upregulation of antiapoptotic and prosurvival genes and reciprocal downregulation of the genes regulating the apoptosis pathway in ectopic endometrial cells[48].

\section{IL-17A Contributes to the Pathogenesis of Endometriosis by Triggering Proinflammatory Cytokines and Angiogenic Growth Factors}

The data show a differential expression of IL-17A in human ectopic endometriotic lesions and matched eutopic endometrium from women with endometriosis. Importantly, surgical removal of lesions resulted in significantly reduced plasma IL-17A concentrations. [49].

Immunohistochemistry revealed localization of IL$17 \mathrm{~A}$ primarily in the stroma of matched ectopic and eutopic tissue samples. In vitro stimulation of endometrial epithelial carcinoma cells, Ishikawa cells, and HUVECs with IL-17A revealed significant increase in angiogenic (vascular endothelial growth factor and IL-8), proinflammatory (IL-6 and IL-1 $\beta$ ), and chemotactic cytokines (G-CSF, CXCL12, CXCL1, and CX3CL1)[49].

Furthermore, IL-17A promoted tubulogenesis of HUVECs plated on Matrigel in a dose-dependent manner. Thus, we provide the first evidence, to our knowledge, that endometriotic lesions produce IL$17 \mathrm{~A}$ and that the removal of the lesion via laparoscopic surgery leads to the significant reduction in the systemic levels of IL-17A. Taken together, our data show a likely important role of IL-17A in promoting angiogenesis and proinflammatory environment in the peritoneal cavity for the establishment and maintenance of endometriosis lesions.[49].

\section{Genetics}

Over the past decade several authors have employed gene arrays to identify endometriosis related genes. Using laser capture microdissection and high throughput and high resolution comparative genomic hybridization $(\mathrm{CGH})$ arrays, considerable genomic alterations in both eutopic and ectopic endometria of women with endometriosis have been identified [50] [51].

Individual genomic changes (found by genotyping including genome-wide association studies) that have been associated with endometriosis include:

Changes on chromosome 1 near WNT4[52]Changes on chromosome 2 near GREB1]52].

Changes on chromosome 6 near ID4[52]

Changes on chromosome 7 in the 7 p15.2 region.[52][53]

Changes on chromosome 9 near CDKN2BAS.[52].

Changes on chromosome 10 at region 10q26.[54]

Changes on chromosome 12 near VEZT.[52]. 


\section{Stem cell}

Human endometrium is a dynamic organ that normally undergoes repetitive cyclic regeneration. To enable this rapid regeneration, it is not surprising that the endometrium contains a reservoir of progenitor stem cells. However, this pool of cells that allows the growth of the endometrium also allows for unrestrained growth that can reach beyond the endometrium.[55].

Recent characterization of stem cell populations within human endometrium has opened the possibility of understanding their physiologic as well as their pathologic roles. While stem cells are critical to the cyclic regeneration of a healthy endometrium, we have shown that both endometrium-derived and bone marrow-derived stem cells can migrate to ectopic sites and contribute to the development of endometriosis[55]. Furthermore, endometriosis interferes with the normal stem cell trafficking to the uterus that is necessary for endometrial growth and repair. Altered stem cell mobility and engraftment characterize this disease[55].

\section{Discussion}

The different theories implicated in the pathogenesis of endometriosis indicate that the aetiology of endometriosis is complex and multifactorial, involving hormonal, genetic, immune, and environmental components.

While retrograde menstruation may be one of the initiating steps in the pathogenesis of superficial endometriosis, genetic and microenvironmental factors that prevent clearance of ectopic lesions and allows remodelling of peritoneum are essential for the propagation of endometriotic lesions[56]

\section{Conclusion}

Ectopically placed stem cells that are of endometrial or haematopoietic origin or abnormal endometrial differentiation of a resident tissue stem cell may be the first step in the establishment of an ectopic endometrial lesion. The subsequent proliferation and propagation of such lesions may also be dependent on mobile, endometrial progenitor-type cells in these ectopic lesions that are involved in initiating further lesion and also in maintaining the disease. A dysfunctional immune clearance and a genetic predisposition that allow these ectopic lesions to grow in an aberrant microenvironment may also contribute to the development of the disease.

The current therapeutic regimens for endometriosis are usually based on manipulating the ovarian steroid hormones that may preferentially target terminallydifferentiated ectopic endometriotic cells which would normally die off via apoptosis, while the stem cells that propagate the disease may not be affected. Improving our understanding of the pathogenesis of endometriosis will direct further future work on more appropriate therapeutic targets that can provide the much needed curative and universally acceptable treatments for endometriosis.

\section{$\underline{\text { References }}$}

1. Giudice C. Endometriosis. The New England Journal of Medicine. 2010; 362(25):2389-2398.

2. Carneiro MM, Filogônio ID, Costa LM, De Ávila I, and Ferreira MC. Accuracy of clinical signs and symptoms in the diagnosis of endometriosis. Journal of Endometriosis. 2010; 2(2):63-70.

3. Burney RO and Giudice L. Pathogenesis and pathophysiology of endometriosis. Fertility and Sterility. 2012; 98: 511-519.

4. Pritts EA and Taylor RN. An evidence-based evaluation of endometriosis-associated infertility. Endocrinology and Metabolism Clinics of North America. 2003; 32(3):653-667.

5. Sharpe-Timms KL. Defining endometrial cells: the need for improved identification at ectopic sites and characterization in eutopic sites for developing novel methods of management for endometriosis. Fertility and Sterility. 2005; 84(1): 35-37.

6. Canis.M, Donnez JG, Guzick DS. et al. Revised American Society for reproductive medicine classification of endometriosis: 1996. Fertility and Sterility. 1997; 67(5): 817-821,.

7. Adamson GD. Endometriosis classification: an update. Current Opinion in Obstetrics and Gynecology. 2011; 23(4): 213-220.

8. Fedele L, Bianchi S, Zanconato G, Raffaelli R, and Berlanda $\mathrm{N}$. Is rectovaginal endometriosis a progressive disease? American Journal of Obstetrics and Gynecology. 2004;191(5):1539-1542.

9. Nassif J, Mattar S, Abu Musa A, and Eid A. Endometriosis and cancer: what do we know? Minerva Ginecologica. 2013; 65:167-179.

10. Vinatier D., Orazi G., Cosson M., Dufour P. Theories of endometriosis.European Journal of Obstetrics \& Gynecology and Reproductive Biology. May2001;96(1): 21-34.

11. Bulletti C, Coccia ME, Battistoni S, Borini A Endometriosis and infertility. J. Assist. Reprod. Genet. August 2010; 27 (8): 441-7. 
12. McGrath, Patrick J.; Stevens, Bonnie J.; Walker, Suellen M.etal. Oxford Textbook of Paediatric Pain. OUP Oxford; 2013: 300.

13. GBD 2013 Mortality and Causes of Death Collaborators (17 December 2014). Global, regional, and national age-sex specific all-cause and cause-specific mortality for 240 causes of death, 1990-2013: a systematic analysis for the Global Burden of Disease Study. Lancet. 2013; 385: 117-71.

14. Brosens I. Endometriosis: Science and Practice. John Wiley \& Sons; 2012: 3..

15. Batt, Ronald E. . A history of endometriosis. London: Springe; 2011: 13-38..

16. Nezhat C, Nezhat F, Nezhat C .Endometriosis: ancient disease, ancient treatments. Fertility and Sterility. December 2012; 98 (6): S1-62.

17. Aghajanova L. and Giudice LC.Molecular evidence for differences in endometrium in severe versus mild endometriosis. Reproductive Sciences. 2011;18(3):229-251.

18. Cornillie FJ, Oosterlynck D,Lauweryns JM, and Koninckx PR. Deeply infiltrating pelvic endometriosis: histology and clinical significance. Fertility and Sterility. 1990;53(6):978-983.

19. SamerSourial, Nicola Tempest, DharaniK,Hapangama. Theories on the Pathogenesis of Endometriosis. International Journal of ReproductiveMedicine.2014; 2014) ArticleID 179515: 9.

20. Fauser BC, Diedrich K, Bouchard P, Domínguez F, Matzuk M, Franks S etal .Contemporary genetic technologies and female reproduction. Hum. Reprod. Update. 2011; 17 (6): 829-47.

21. Sasson IE and Taylor HS. Stem cells and the pathogenesis of endometriosis. Annals of the New York Academy of Sciences. 2008; 1127:106-115.

22. Harirchian P, Gashaw I, Lipskind ST et al. Lesion kinetics in a non-human primate model of endometriosis. Human Reproduction. 2012; 27 : 2341-2351.

23. D'Hooghe TM, Bambra CS, Suleman MA, Dunselman GA, Evers HL, and Koninckx PR. Development of a model of retrograde menstruation in baboons (Papio anubis). Fertility and Sterility. 1994;62(3):635-638.

24. Dehoux JP, Defrre S, Squifflet J et al. Is the baboon model appropriate for endometriosis studies? Fertility and Sterility.2011; 96(3):728-e3.

25. Gleicher N, el-Roeiy A, Confino E, Friberg J .Is endometriosis an autoimmune disease? Obstet Gynecol. 1987;70 (1): 115-22.
26. Capellino S, Montagna P, Villaggio B, Sulli A, Soldano $\mathrm{S}$, et al. Role of estrogens in inflammatory response: expression of estrogen receptors in peritoneal fluid macrophages from endometriosis. Annals of the New York Academy of Sciences. June 2006; 1069: 263-7.

27. Brosens I.and Benagiano G. Is neonatal uterine bleeding involved in the pathogenesis of endometriosis as a source of stem cells? Fertility and Sterility. 2013; 100:622-623.

28. Gruenwald P. Origin of endometriosis from the mesenchyme of the celomic walls. American Journal of Obstetrics and Gynecology. 1942; 44(3): $470-474$.

29. Caroline W. Diagnosis and Treatment of Endometriosis. American Academy of Family Physicians. 1999 Oct 15;60(6):1753-1762.

30. Figueira PG, Abrão MS, Krikun G, and Taylor H. Stem cells in endometrium and their role in the pathogenesis of endometriosis. Annals of the New York Academy of Sciences. 2011;1221(1)10-17.

31. Signorile PG, Baldi F, Bussani R, D'Armiento M, De Falco M, Baldi A. Ectopic endometrium in human foetuses is a common event and sustains the theory of müllerianosis in the pathogenesis of endometriosis, a disease that predisposes to cancer. Journal of Experimental \& Clinical Cancer Research. April 2009; 28: 49.

32. Bruner-Tran KL, Yeaman GR , Crispens MA., Igarashi TM, Osteen KG. Dioxin may promote inflammation-related development of endometriosis. Fertility and Sterility. 2008; 89: 1287-98.

33. Laschke MW, Giebels C, Menger MD. Vasculogenesis: a new piece of the endometriosis puzzle. Hum.Reprod.Update. 2011;17(5):628-36.

34. Carmeliet P. Mechanisms of angiogenesis and arteriogenesis. Nat Med. 2000;6:389-395..

35. Kässmeyer S, Plendl J, Custodis P, Bahramsoltani M. New insights in vascular development: vasculogenesis and endothelial progenitor cells. Anat Histol Embryol. 2009;38:1-11.

36. Murasawa S, Asahara T. Endothelial progenitor cells for vasculogenesis. Physiology (Bethesda) . 2005;20:36-42.

37. Morotti M, Vincent K, Brawn J, Zondervan KT, Becker CM. Peripheral changes in endometriosisassociated pain. Human Reproduction Update. 2014; 20 (5): 717-736.7.

38. Parente Barbosa C, Bentes De Souza AM, Bianco $\mathrm{B}$, and Christofolini DM. The effect of hormones on endometriosis development. Minerva Ginecologica .2011; 63(4):375-386. 
39. Murphy AA, Palinski W, Rankin S, Morales AJ, and Parthasarathy S. Evidence for oxidatively modified lipid-protein complexes in endometrium and endometriosis. Fertility and Sterility. 1998; 69(6):1092-1094.

40. Wang Y, Goldberg J, Sharma RK, Agarwal A, and Falcone T. Importance of reactive oxygen species in the peritoneal fluid of women with endometriosis or idiopathic infertility. Fertility and Sterility. 1997; 68(5)826-830,

41. Kumar S and Bandyopadhyay U. Free heme toxicity and its detoxification systems in human. Toxicology Letters. 2005; 157(3):175-188.

42. Gupta S, Agarwal A, Krajcir N, andAlvarez JG. Role of oxidative stress in endometriosis. Reproductive BioMedicine Online. 2006;13(1):126-134.

43. Yuk JS, Park EJ, Seo YS, Kim HJ, Kwon SY, Park WI. Graves Disease Is Associated With Endometriosis: A 3-Year Population-Based CrossSectional Study. Medicine (Baltimore). 2016 Mar;95(10):e2975.

44. Sikora J, Mielczarek-Palacz A, and KonderaAnasz Z. Role of Natural Killer cell activity in the pathogenesis of endometriosis. Current Medicinal Chemistry. 2011; 18(2):200-208.

45. Osuga Y., Koga K, Hirota Y, Hirata T, Yoshino O, and Taketani Y. Lymphocytes in Endometriosis. American Journal of Reproductive Immunology. 2011; 65(1)1-10.

46. McLaren J, Prentice A,. Charnock-Jones DS, and Smith SK. Vascular endothelial growth factor (VEGF) concentrations are elevated in peritoneal fluid of women with endometriosis. Human Reproduction. 1996; 11(1): 220-223.

47. Hapangama DK, Turner MA, Drury J et al. Aberrant expression of regulators of cell-fate found in eutopic endometrium is found in matched ectopic endometrium among women and in a baboon model of endometriosis. Human Reproduction. 2010; 25(11):2840-2850.

48. Ferryman SR and Rollason TP. Pathology of the uterine body. Current Opinion in Obstetrics and Gynecology. 1994; 6(4)344-350.

49. Soo HA, Andrew KE, Sukhbir SS, Steven LY, Bruce AL and Chandrakant T. IL-17A Contributes to the Pathogenesis of Endometriosis by Triggering Proinflammatory Cytokines and Angiogenic Growth Factors. The Journal of Immunology. 2015; 195 (6):2591-2600.

50. Afshar Y, Hastings J, Roqueiro D, Jeong JW, Giudice LC, and Fazlebas AT. Changes in eutopic endometrial gene expression during the progression of experimental endometriosis in the baboon, papio Anubis. Biology of Reproduction. 2013; 88: 44.

51. Khan MA, Sengupta J, Mittal S, and Ghosh D. Genome-wide expressions in autologous eutopic and ectopic endometrium of fertile women with endoemtriosis. Reproductive Biology and Endocrinology. 2012;10: 84.

52. Rahmioglu N, Nyholt DR, Morris AP, et al. . Genetic variants underlying risk of endometriosis: insights from meta-analysis of eight genomewide association and replication datasets. Human Reproduction Update. 2014; 20 (5): 702-716.

53. Painter JN, Anderson CA, Nyholt DR, et al. . Genome-wide association study identifies a locus at 7 p15.2 associated with endometriosis. Nature Genetics. January 2011; 43 (1): 51-4.

54. Treloar SA, Wicks J, Nyholt DR, et al. Genomewide linkage study in 1,176 affected sister pair families identifies a significant susceptibility locus for endometriosis on chromosome 10q26. American Journal of Human Genetics. September 2005; 77 (3): 365-76.

55. Hufnagel D, Li F, Cosar E, Krikun G, Taylor HS. The Role of Stem Cells in the Etiology and Pathophysiology of Endometriosi. Seminars in reproductive medicine. September 2015; 33 (5): 333-40.

56. Du H. and Taylor HS. Reviews: stem cells and female reproduction. Reproductive Sciences. 2009; 16(2):126-139. 